\title{
sciendo
}

\section{Changes in Floor Exercise Characteristics in World Elite Male Gymnasts}

\author{
by \\ Jonas Rohleder ${ }^{1}$, Tobias Vogt ${ }^{1}$
}

\begin{abstract}
In artistic gymnastics, athletes need to compose their floor exercise routines in accordance with the Code of Points which is provided by the International Gymnastics Federation. In view of the latest rule modifications subsequent to the 2016 Olympic Games, this study investigates recent changes in judges' scorings with respect to changes in the characteristics of floor exercise routines in world elite male gymnasts. Therefore, all floor exercise routines $(n=25)$ performed in the men's floor exercise finals at the World Championships in 2013 (WC13), 2015 (WC15) and 2017 (WC17) were examined using video analysis. Gymnasts' scores (difficulty, execution and final scores), element group distributions and further exercise characteristics (e.g., the total amount of somersaults, twists, and landing errors) were defined as variables. Decreases in difficulty and execution scores were revealed for WC17 compared to WC15 and WC13, respectively $(p<.01)$. Additionally, a decrease in the number of backward jumped elements was observed at WC17 $(p<.01)$, whereas the number of forward jumped elements increased $(p<.01)$. Furthermore, a significantly increased number of landing errors $(p<.05)$ negatively correlated with the decrease in execution $(p<.001)$ and final scores $(p<.05)$. To conclude, current compositional trends in men's floor exercise encourage to include difficult forward jumps and multiple twisting connections in consideration of prudent teaching with respect to the gymnasts' individual abilities and the decisive influence of stick landings.
\end{abstract}

Key words: gymnastics, performance, composition, landings, competition, judging.

\section{Introduction}

In men's artistic gymnastics, floor exercise is one out of six events for gymnasts to compete in the all-around competition. In official competitions such as the European or World Championships the best eight gymnasts on each apparatus may qualify for the single event finals, for example floor exercise. In general, wellbalanced routines on floor exercise are predominantly composed of several acrobatic somersault and twisting elements accompanied by non-acrobatic elements that need to meet several requirements of strength, balance or flexibility (FIG, 2017). However, all gymnasts have to adapt their routines to the currently valid Code of Points (CoP) which is provided and revised at regular intervals after each Olympic Games by the International Gymnastics
Federation (FIG). On the one hand, the CoP is the guideline for gymnastic coaches and gymnasts for composing their routines in preparation for competitions. On the other hand, the $\mathrm{CoP}$ assures objective and standardised judging of gymnastics exercises with the aim to identify the best gymnasts in any competitions (FIG, 2017; Pizzera, 2012). Previous reports accentuate satisfactory reliability and validity in gymnastics judging (e.g., Čuk et al., 2012; Dallas and Kirialanis, 2010; FIG, 2017). However, there is evidence that gymnastics' performance assessments cannot unexceptionally be based on objective criteria, but are also influenced by, for example, subjective perceptions and differences in gaze behaviour (Bard et al., 1980; Ste-Marie, 2000), sport-specific and judging experience (Heinen et al., 2012;

1 - Institute of Professional Sport Education and Sport Qualifications, German Sport University Cologne, Cologne, Germany. 
Pizzerra, 2012; Plessner and Schallies, 2005), and the mutual influence of the judges and, thus, bias in judging performances (Boen et al., 2008; Leskošek et al., 2010, 2012; Plessner, 1999).

After completing their performance, all gymnasts receive a final score (F-score) serving as evaluation of the performed routine. The F-score is determined and published by the judges panel and decides the ranking of the participants in gymnastics competitions. As a consequence of a fundamental revision of the CoP in 2005, to date the F-Score is calculated by the addition of two fundamental components comprising the score for the difficulty (D-score) and the score for the execution quality (E-score) of the presented routines (FIG, 2017). Additionally, specific penalties may be applied by the D-Jury to the FScore in case of, for example, landings outside of the $12 \times 12 \mathrm{~m}$ floor area (FIG, 2017). In the past decade, research on gymnastic judging has emphasised the dominance of the difficulty compared to the performances' execution quality (Čuk and Forbes, 2010; Leskošek et al., 2013). However, with the objective of equivalent importance of both components, the $\mathrm{CoP}$ provides detailed criteria for the independently judging Dand E-Jury to assess the gymnasts' routines (FIG, 2017).

Unaffected by any rule adjustments since 2006, to date the D-score is, inter alia, determined by the additional difficulty value of the ten most difficult elements performed within the routine (FIG, 2017). Therefore, each admitted gymnastics element is assigned a level of difficulty within a range of A-value (= 0.1 points) until I-value ( $=0.9$ points) (FIG, 2017). Furthermore, the gymnast has to include at least one element from several specific element groups in order to assure performance versatility. Each fulfilled element group awards 0.5 points for the D-score (FIG, 2017). Recent modifications of the CoP define three different element groups on floor exercise that require fulfilment within the ten counting elements for the D-score, I: Non-acrobatic Elements (EG1); II: Acrobatic elements forward (i.e., forward jumped elements, EG2); III: Acrobatic elements backward (i.e., backward jumped elements, EG3). Over the last Olympic cycles including the 2016 Olympic Games, even four element groups were demanded on floor exercise inclusive of EG4: Acrobatic elements sideways and backward jumped elements with half turns and forward somersaults (= Arabiantype elements with or without rollout), respectively (FIG, 2013). In the course of the latest reform of the CoP in 2017, all acrobatic elements sideways were prohibited and, besides, all Arabian-type elements were included to the backward jumped elements (EG3) (FIG, 2017). Thus, in modern floor exercise performances, one less element group is now recognised for the Dscore calculation, whilst five elements (previously: four elements) of each element group may be recognised for the additional difficulty value (FIG, 2013; FIG, 2017). It has to be taken into consideration that difficult EG2- and EG3elements including multiple somersault rotations have been upgraded in the current version of the CoP (FIG, 2017). Moreover, rules concerning the awarding of immediate connections (D or higher + $\mathrm{B}$ or $\mathrm{C}=+0.1$; $\mathrm{D}$ or higher $+\mathrm{D}$ or higher $=+0.2$ ) were recently modified (FIG, 2017). While the total amount of awarded connections was previously unlimited, it seems important to note that to date there is a limit of two connections in total in one routine that may be awarded with additional points. Considering that simple twisting elements were downgraded, requirements for awarded connections of acrobatic jumps were impeded with the new CoP.

The E-score represents the execution quality of the gymnast's performance (Čuk and Forbes, 2010; Pizzera, 2012). The E-score is determined by a baseline of 10.0 points, provided in order to have a gymnast perform at least seven elements (FIG, 2017). Starting from this basis, the execution quality is evaluated by deductions concerning aesthetic, technical and compositional errors applied in tenths of a point (FIG, 2017). Any deviations from the expected technical and aesthetic perfection result in deductions for small (0.1 points), medium (0.3 points) or large errors (0.5 points) or even falls (1.0 points), respectively (FIG, 2017). It is well-accepted that the quality of the landing followed by acrobatic elements is one of the most important factors for determining the E-score (Marinšek and Čuk, 2010). Considering that all somersault movements finishing with a rollout and jumps to the prone position were prohibited in the currently valid CoP (FIG, 2017), there are no alternatives to stick landings anymore facilitating prevention from landing 
errors like steps or hops. Furthermore, Marinšek and Čuk (2010) showed that different characteristics of the flight phase (e.g., the axis of rotation, the number of somersaults and twists and the initial landing height) affected the quality of a landing. It was further reported that multiple twisting somersaults put the highest demands on the landing in order to achieve still equilibrium (Marinšek and Čuk, 2010). With respect to increasing D-scores within the first two Olympic cycles since the CoP was revised in 2006, the Escore's subordinate role in differentiating the level of performance was well documented (Čuk and Forbes, 2010; Leskošek et al., 2013).

Reviewing the latest rule changes in the $\mathrm{CoP}$ with reference to current analyses on changes in elite gymnastics' scores, research merely addressed the weight of difficulty and execution components (e.g., Čuk and Forbes, 2010; Leskošek et al., 2013) as well as influential aspects impairing or improving judgement performances (e.g., Boen et al., 2008; Dallas and Kirialanis, 2010; Pizzera, 2012). In consideration of implications regarding the application of the periodic rule adaptions, investigations on exercise characteristics (i.e., difficulty and execution quality) influencing judges' scoring in world elite gymnastics performances remain, at least in parts, to be elucidated with regard to progressions in current floor exercise performances.

Therefore, the purpose of this study was to examine and quantify general changes in floor exercise characteristics in men's world elite gymnasts in consideration of the latest modifications in the CoP 2017. In view of recent changes regarding the element group elimination and impeded connection awarding (FIG, 2013; FIG, 2017), it was hypothesised that (1) the gymnasts' scorings showed decreased D-scores in 2017. (2) Decreases were further hypothesised for E-scores with respect to presumably increased landing errors due to the definitive prohibition of rollout somersault elements and, thus, absent options to avoid stick landings (Čuk and Forbes, 2010; Leskošek et al., 2013; Marinšek and Čuk, 2010). (3) EG3 was hypothesised to increase the total amount of recognised elements for the Dscore which was attributed to the increased maximum of five elements for the same element group and the inclusion of Arabian-type elements into EG3 (FIG, 2013; FIG, 2017).

\section{Methods}

\section{Participants and Design}

In order to investigate changes in floor exercise characteristics within chronological time intervals of two years, the men's floor exercise finals of the artistic gymnastics World Championships in 2013 in Antwerp (WC13) and in 2015 in Glasgow (WC15) were compared to the finals of the first World Championships after recent rule adaptions in Montreal in 2017 (WC17). Therefore, all floor exercise routines $(n=25)$ performed during the men's floor finals at WC13, WC15 and WC17 were analysed. Eight gymnasts competed in each floor exercise final of WC13 and WC15, whereas even nine gymnasts took part in the WC17 finals due to a successful protest by one gymnast during the qualifications. At the time of the competitions, all floor finalists were at least sixteen years of age. This study was approved by the university's ethical committee of the German Sport University Cologne, thus, the material in the manuscript has been acquired according to the ethical standards.

\section{Measures and Procedures}

To evaluate changes in judging and the scores' level with respect to performance-related floor exercise characteristics, the following variables were defined for further analyses:

- Judges' scores (i.e., difficulty score $=$ Dscore; execution score = E-score; and final score $=$ F-score)

- Element groups (i.e., number of nonacrobatic elements = EG1; number of forward jumped elements = EG2; number of backward jumped elements $=$ EG3)

- Performance characteristics (i.e., the total amount of somersault rotations, twisting rotations and acrobatic lines; the percentage of stick landings; the total amount of landing errors which were defined by any steps and hops; the total amount of awarded connection points and highly difficult acrobatic connections of two D-value elements or higher)

- With respect to only one existing case of penalty within the sample size (applied at WC15), the present approach completely excluded analyses regarding penalty deductions.

For each competition, the gymnasts' scores given by the judges' panels were received 
from the official websites of the WC13, WC15 and WC17 where all scores are open to public inspection. Furthermore, the floor exercise routines of all finalists within the three analysed World Championships are available on the YouTube-'FIG Channel'. Thus, the element groups of the ten elements counting for the difficulty score as well as further descriptive performance characteristics were determined with help of video analyses.

\section{Statistical Analysis}

For all variables (judges scores: D-Score, E-Score; F-Score; element groups: EG1, EG2; EG3; performance characteristics: number of somersaults, twists, acrobatic lines; stick landings; landing errors; connection points; D+Dconnections), non-parametric multiple comparisons were calculated using the KruskalWallis test to determine changes within the factor time of measurement (WC13, WC15 and WC17). In case of obtained significant effects, pairwise comparisons were computed post hoc in accordance to Bonferroni adjustments. The Eta Square $\left(\eta^{2}\right)$ was used to identify effects from the Kruskal-Wallis analysis, whereas Cohen's D (d) effect sizes were calculated to interpret subsequent pairwise comparisons (Cohen, 1992). Furthermore, possible correlations between judges' scores and performance characteristics were determined using the Pearson's correlation coefficient. Statistical analyses were performed using SPSS for Windows (version 22.0). Figures and tables were generated using the Microsoft Excel software (version 2013). The statistics are accompanied by $95 \%$ confidence intervals ( $95 \%$ $\mathrm{CI})$, whereas data in the figures and tables are presented as mean $(\mathrm{M}) \pm$ standard deviation (SD). The level of significance was set at $p<.05$.

\section{Results}

\section{Judges' scores}

The Kruskal-Wallis test revealed significant differences for D-scores, $\chi^{2}=9.698, p<$ $.01, \eta^{2}=.40,95 \%$ CI $[6.42,6.77]$, E-scores, $\chi^{2}=$ $10.175, p<.01, \eta^{2}=.42,95 \%$ CI $[8.19,8.55]$, and Fscores, $\chi^{2}=11.696, p<.01, \eta^{2}=.49,95 \%$ CI [14.68, 15.24].

Post hoc analysis revealed significant decreases for D-scores between WC15 and WC17, $p<.01, d=1.49$, whereas D-scores, at least, decreased by trend between WC13 and WC17, $p<.10, d=1.11$. Furthermore, post hoc analysis obtained significant decreases in E-scores, $p<.01$, $d=1.85$, and F-scores, $p<.01, d=2.18$, between WC13 and WC17, whereas F-scores, at least, decreased by trend between WC15 and WC17, $p=$ $.06, d=1.60$ (Figure 1A; Table 1).

\section{Element groups}

The Kruskal-Wallis test showed no differences for EG1, $\chi^{2}=.315, p=.85, \eta^{2}=.01,95 \%$ CI [1.03, 1.53]. However, significant differences were obtained for EG2, $\chi^{2}=11.288, p<.01, \eta^{2}=.47$, $95 \%$ CI $[3.42,4.10]$, and EG3, $\chi^{2}=11.985, p<.01, \eta^{2}$ $=.50,95 \% \mathrm{CI}[4.63,5.29]$.

Post hoc analysis revealed significant increases for EG2 between WC13 and WC17, $p<$ $.01, d=1.89$, whereas EG2 increased, at least by trend, between WC15 and WC17, $p=.08, d=1.54$. Furthermore, post hoc analysis obtained significant decreases for EG3 between WC13 and WC17, $p<$ $.01, d=2.09$ (Figure 1B; Table 1).

\section{Performance characteristics}

The Kruskal-Wallis test showed no differences for the total amount of somersaults, $\chi^{2}$ $=.479, p=.79, \eta^{2}=.02,95 \%$ CI [10.67, 11.91], twists, $\chi^{2}=1.554, p=.46, \eta^{2}=.07,95 \%$ CI $[13.16,17.00]$, acrobatic lines, $\chi^{2}=1.417, p=.49, \eta^{2}=.06,95 \% \mathrm{CI}$ $[5.83,6.17]$ stick landings, $\chi^{2}=1.616, p=.47, \eta^{2}=$ $.07,95 \%$ CI $[18.26,38.22]$, general connection points, $\chi^{2}=4.292, p=.12, \eta^{2}=.18,95 \%$ CI $[.28, .36]$ and highly difficult acrobatic connections of two D-value elements or higher, $\chi^{2}=1.672, p=.43, \eta^{2}=$ $.07,95 \%$ CI $[.29, .83]$. However, significant differences were obtained for the total amount of landing errors, $\chi^{2}=7.313, p<.05, \eta^{2}=.31,95 \% \mathrm{CI}$ $[3.66,5.22]$.

Post hoc analysis revealed significant increases for landing errors between WC13 and WC17, $p<.05, d=1.44$, (Figure 2A; Table 1).

Correlations

The correlation analysis revealed significant negative correlations between the E-score and landing errors, $\mathrm{r}(23)=-.68, p<.001$, and between the F-score and landing errors, $\mathrm{r}(23)=-.47, p<.05$ (Figure 2B). There were no correlations between landing errors and the total amount of twists, $\mathrm{r}(23)=.02, p=.92$. 


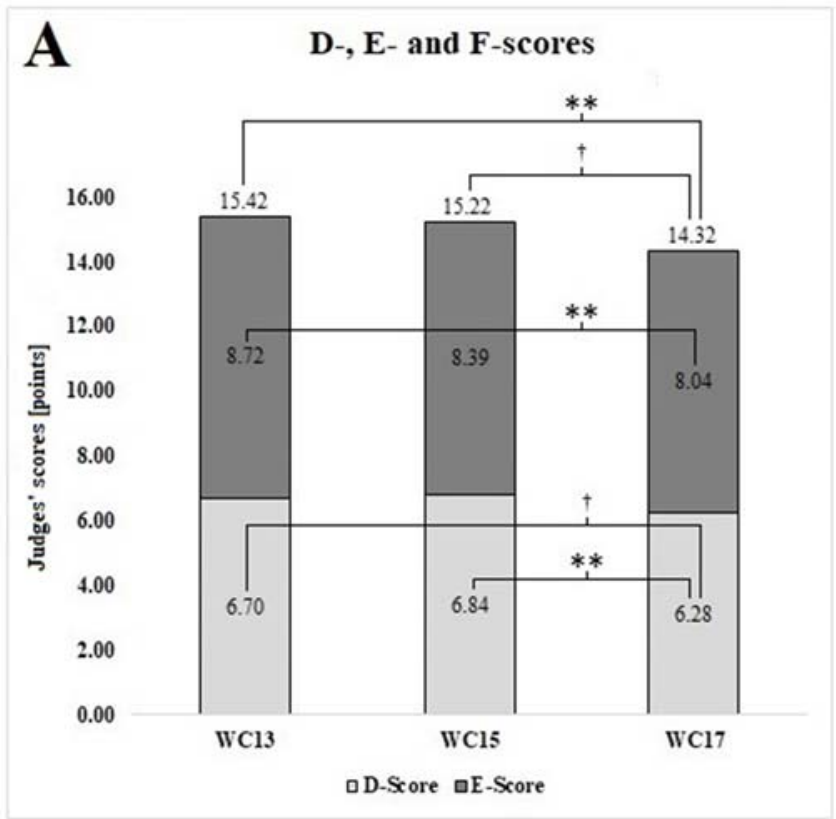

\section{B Element groups' distribution}

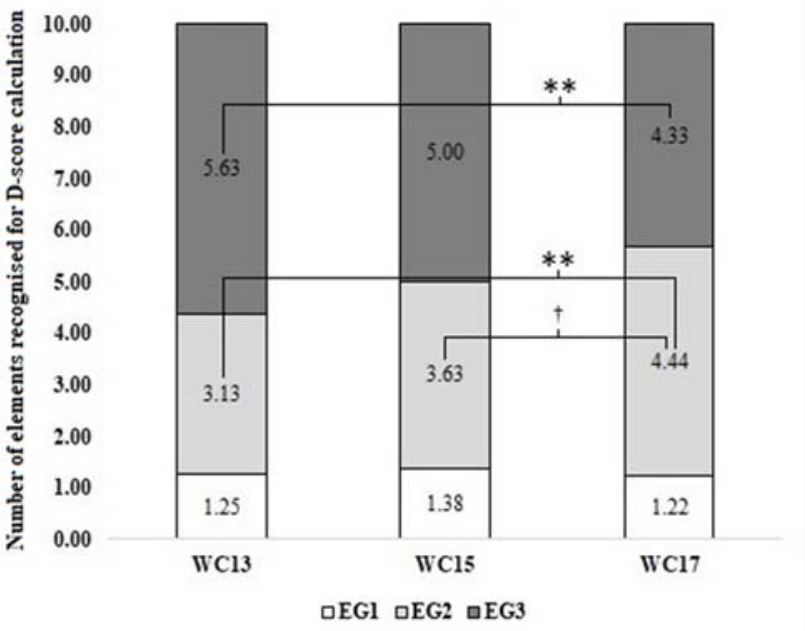

Figure 1

A: Gymnasts' mean F-scores excluding penalties, separated by mean scores for Difficulty (D-score) and Execution (E-score) during the World Championships in 2013 (WC13), 2015 (WC15) and 2017 (WC17); B: Mean numbers of elements within the non-acrobatic elements (EG1), the forward jumped elements (EG2) and the backward jumped elements (EG3) counted for the gymnasts' difficulty score during WC13, WC15 and WC17. Levels of significance (Bonferroni-adjusted): ${ }^{* *} p<.01 ;{ }^{*} p<.05 ;{ }^{*} p<.10$ 

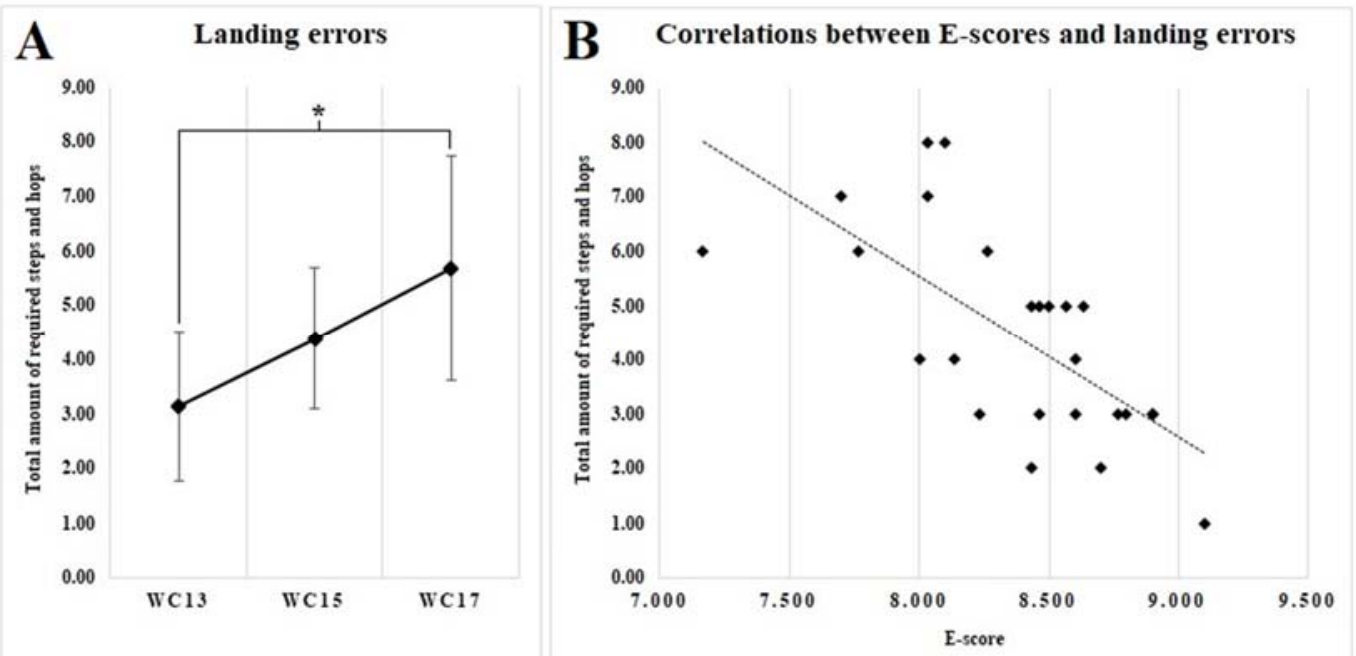

\section{Figure 2}

A: Gymnasts' mean number of landing errors (including any steps and hops) during the World Championships in 2013 (WC13), 2015 (WC15) and 2017 (WC17). Levels of significance (Bonferroni-adjusted): ${ }^{* *} p<.01 ;{ }^{*} p<.05 ;{ }^{*} p<.10 ; B$ : Negative correlations between the gymnasts' $E$-scores and the mean number of landing errors (including any steps and hops).

Table 1

Changes in male floor exercise characteristics during the World Championships in 2013, 2015 and 2017.

\begin{tabular}{lllll}
\hline & Variables & $\begin{array}{l}\text { WC13 } \\
(\mathrm{M} \pm \mathrm{SD})\end{array}$ & $\begin{array}{l}\text { WC15 } \\
(\mathrm{M} \pm \mathrm{SD})\end{array}$ & $\begin{array}{l}\text { WC17 } \\
(\mathrm{M} \pm \mathrm{SD})\end{array}$ \\
\hline Judges scores & D-score & $6.70 \pm 0.34$ & $6.84 \pm 0.33$ & $6.28 \pm 0.41$ \\
& E-score & $8.72 \pm 0.24$ & $8.39 \pm 0.29$ & $8.04 \pm 0.45$ \\
& F-score & $15.42 \pm 0.33$ & $15.22 \pm 0.49$ & $14.32 \pm 0.62$ \\
Element group & EG1 & $1.25 \pm 0.71$ & $1.38 \pm 0.74$ & $1.22 \pm 0.44$ \\
& EG2 & $3.13 \pm 0.84$ & $3.63 \pm 0.52$ & $4.44 \pm 0.53$ \\
Performance & Somersaults & $5.63 \pm 0.74$ & $5.00 \pm 0.54$ & $4.33 \pm 0.50$ \\
& Twists & $11.47 \pm 1.40$ & $11.44 \pm 1.64$ & $11.00 \pm 1.58$ \\
& Lines & $13.66 \pm 4.54$ & $15.09 \pm 4.56$ & $16.33 \pm 5.01$ \\
& Stick landings & $5.88 \pm 0.35$ & $6.00 \pm 0.54$ & $6.11 \pm 0.33$ \\
& (percentage) & $35.63 \pm 26.92$ & $26.50 \pm 19.26$ & $22.11 \pm 25.06$ \\
& Landing errors & $3.13 \pm 1.36$ & $4.38 \pm 1.30$ & $5.67 \pm 2.06$ \\
& Connection points & $0.35 \pm 0.12$ & $0.34 \pm 0.09$ & $0.27 \pm 0.05$ \\
& D+D connections & $0.63 \pm 0.74$ & $0.38 \pm 0.74$ & $0.67 \pm 0.50$ \\
& $\begin{array}{l}\text { gymnasts with D+D } \\
\text { (percentage) }\end{array}$ & 50.00 & 25.00 & 66.60 \\
\hline
\end{tabular}

Changes in male floor exercise characteristics during the World Championships in 2013, 2015 and 2017. 


\section{Discussion}

The present study aimed to examine and quantify general changes in floor exercise characteristics in men's world elite gymnasts in consideration of the latest modifications in the CoP 2017. Main findings were decreased judges' scores and an increased amount of landing errors. Moreover, an increased number of forward jumped acrobatic elements as opposed to a decreased number of backward jumped elements was obtained.

The decreased D-scores that were observed at WC17 compared to WC15 and, at least by trend, WC13 support our first hypothesis (1). With respect to recent changes in the CoP regarding the elimination of one element group (FIG, 2017), we primarily attribute the observed decreases between WC15 and WC17 to the missing half point that was previously awarded for the presentation of acrobatic elements sideways or Arabian-type elements (EG4) within the last Olympic cycles (FIG, 2013). This assumption is confirmed by the observation that decline in the D-score at WC17 compared to WC15 amounted to approximately half a point. However, differences in D-scores failed significance between WC13 and WC17. With respect to this, it seems reasonable that these findings presumably result from general difficulty-related progress in floor exercise during the last Olympic cycle and, thus, between WC13 and WC17. Although there were no differences for the total amount of somersault rotations and acrobatic lines, the continuously increased number of twists that were integrated into the somersault movements during the three World Championships' floor finals may, at least in parts, support this interpretative approach and may contribute to a mean difficulty score difference less than half a point between WC13 and WC17. Additionally, rule changes in awarding immediate connections of acrobatic elements (FIG, 2013; FIG, 2017) may explain current D-score changes. Although failing significance, decreases in awarded connection points were associated with recent limitations for acrobatic connections and, thus, generally decreased D-scores (FIG, 2017). However, it seems important to note that at WC17 $66.6 \%$ (six of nine gymnasts in total) showed highly difficult acrobatic connections (immediate connection of two D-value elements or higher) awarded with 0.2 points, whereas at WC13 and WC15, only $50.0 \%$ (four of eight gymnasts in total at WC13) and $25.0 \%$ (two of eight gymnasts in total at WC15) performed suchlike acrobatic connections, respectively. Thus, with respect to the characteristics of modern floor exercise performances, there are reasons to assume that the difficulty of floor exercise routines generally increased independent of the amount that is indicated by the D-score, which is in line with general observations during the past Olympic cycles (Leskošek et al., 2013).

The declined E-scores that we observed at WC17 compared to WC13 support our second initial hypothesis (2) based on previous reports revealing reduced E-scores in men's artistic gymnastics within the last decade (Čuk and Forbes, 2010; Leskošek et al., 2013). Reduced Escores have to be discussed in relation to landing performances which are suggested to be of utter importance for determining the gymnasts' performances and, thus, rankings in competitions (Marinšek and Čuk, 2010). Only 22.1\% of all landings at WC17 compared to $26.5 \%$ at WC15 and even $35.6 \%$ at WC13 were performed without any steps or hops. These findings further confirm our second hypothesis (2) and are in line with comparable data revealed by Marinšek and Čuk (2010), showing that $29.9 \%$ of somersault landings during the qualifications of the senior Men's European Championships 2004 in Ljubljana were performed into stick landings. However, while a decrease in the percentage of stick landings failed significance, landing errors increased significantly in the form of multiple required steps and hops in order to control equilibrium. Additionally, the larger amount of landing errors may presumably be associated with the elimination of no longer permitted rollout somersault elements (FIG, 2017). Furthermore, and in line with previous reports (Marinšek and Čuk, 2010), negative correlations were revealed for E-scores and landing errors in the present study. Additionally, with respect to increased numbers of twists performed at WC17, it seems reasonable that impaired landing performances may be explained by other findings of Marinšek and Čuk (2010) suggesting most problems in achieving a still equilibrium standing position followed by twisting somersault movements. However, the present study's findings may not confirm this report in view of 
absent correlations between the total amount of landing errors and the number of performed twists. Thus, the impact of landing success on Escores is underlined by present findings suggesting gymnasts and coaches to focus on correct landing techniques, but nevertheless, the impact of multiple rotations around the longitudinal axis on elite gymnasts' landing success or failure remains to be elucidated.

While declined D-scores may presumably be explained by recent CoP modifications, there is, however, reason to believe that decreased Escores cannot be exclusively attributed to an increased amount of landing errors. Thus, although, at least in parts, in line with comparable reports (Marinšek and Čuk, 2010), the present study's findings have to be discussed in relation to divergent reasons. If one takes into consideration that there were no important rule changes in the 2017 CoP concerning execution assessments (as it was already the case since the CoP was reformed in 2006, Leskošek et al., 2013), it seems rational that reduced E-scores are additionally caused by one of the following reasons: (a) difficulty increases may result in overall impaired execution qualities except for landing performances which may seem plausible within the first competition season following rule changes; (b) speculations about reasons for generally declined E-scores may include thoughts concerning changes in rigour regarding the practical application of rules stated in the CoP (FIG, 2013, 2017; Leskošek et al., 2013) which, at best, should be observed and approved by all involved federations in order to progress conformity in worldwide judging (Boen et al., 2008). Besides these pursuing assumptions in addition to discussed findings regarding landing performances, there is no further evidence-based explanation for the causal principles underlying the observed E-score decrease. In consequence, to date the knowledge gap restricts clear statements with regard to the judges' style in applying the CoP whilst assessing elite gymnasts' performances. With respect to aspired practical recommendations for athletes and coaches, it is therefore important to investigate if current Escore decreases in elite gymnasts' floor exercise routines occur due to more detailed and, thus, more frequent deductions by the E-jury (Leskošek et al., 2010, 2013). For this, the present study's findings suggest future research to supplement analyses on gymnasts' performances by continuous analyses on judges' behaviour in terms of, for example, changes in gaze strategies (Bard et al., 1980; Ste-Marie, 2000) or bias in the Epanels' judging (e.g., Boen et al., 2008; Leskošek et al., 2010, 2012; Plessner, 1999) which is fundamentally suggested to be accurate and valid during the last decade (e.g., Dallas and Kirialanis, 2010; Heinen et al., 2012; Leskošek et al., 2010). With this, a comprehensive view on gymnasts' and judges' performances promises extensive insights into expedient exercise adaptions to CoP modifications.

The decreased amount of EG3 elements that was observed at WC17 compared to WC13 contradicts our third hypothesis (3). At first thought, due to the latest rule change that included all Arabian-type elements to the backward jumped elements (FIG, 2017), the present study's findings were expected to obtain an even increased number of EG3 elements. While, in fact, opposite changes occurred at WC17, it seems important to note that the number of EG2 elements increased in WC17 compared to WC13. This provides a reason to believe that recent upgrades in highly difficult forward jumped elements compared to upgraded backward jumped elements have currently been perceived as the more appropriate approach to increase difficulty and, thus, raise floor exercise performances. However, from an economic perspective, it may be questioned whether an increased amount of forward jumped elements has generally been effective to generate enhanced performances. With respect to landing success, Marinšek and Čuk (2010) for example suggest an increase in landing errors followed by forward jumped somersault elements (with and without twists) compared to backward jumped elements. Therefore, future observations and investigations have to monitor the prospects of current forward acrobatics in forthcoming international floor exercise finals.

Taken together, the present study's findings revealed decreased D-scores at WC17 compared to the past Olympic cycle that were primarily caused by substantial rule changes in the modified CoP 2017. Furthermore, scores for execution quality decreased as well which may presumably be associated with impaired landing 
performances. The increased amount of landing errors might be due to current tendencies towards forward jumped elements and increased multiple twisting which are suggested to impede landing preparation.

\section{Limitations}

Despite plausible findings, the small data set resulting from the limited number of eight and nine gymnasts, respectively, competing in the floor exercise finals may be considered as a limitation to generalise the present study's findings. However, the chosen approach offers insights into the world-class routines that have led to international success in the recent past which, thus, justifies the general idea of the present approach. Additionally, it may be argued why this study leaves out the examination of the 2014 World Championships and the 2016 Olympic Games 2016. Nevertheless, taking the special circumstances of Olympic competitions into consideration, the present study's approach which is characterised by a two-year cycle of data analysis was considered as appropriate to provide plausible results. Furthermore, besides the CoP, the limited volume of comparable studies made it difficult to integrate our findings into an appropriate context of literature. Nevertheless, herein a chance was offered to serve specific research purposes that had rather been neglected in international scientific exchange.

\section{Conclusions}

In conclusion, our results provide evidence that current changes in world elite floor exercise gymnasts are characterised by generally decreased score levels due to recent rule changes in the current CoP 2017. With respect to the present study's approach to rule-induced exercise modifications, compositional trends in men's floor exercises encourage elite gymnasts and coaches to invest great efforts in developing difficult forward jumped elements and highly valued twisting connections in consideration of prudent teaching with respect to the gymnasts' individual abilities. While focussing on stick landings is particularly recommended, to date it may not be excluded that current tendencies in composing floor exercise routines lead to increased landing errors and, thus, counteract increased final scores. Therefore, the present approach stresses further research in order to generate expedient practical recommendations regarding floor exercise composing in consideration of the CoP 2017.

\section{References}

Bard C, Fleury M, Carriere L, Halle M. Analysis of gymnastics judges' visual search. Res Q Exercise Sport, 1980; 51(2): 267-273

Boen F, van Hoye K, Auweele Y, Feys J, Smits T. Open feedback in gymnastics judging causes conformity bias based on informational influencing. J Sport Sci, 2008; 26(6): 621-628

Cohen J. A power primer. Psychol Bull, 1992; 112(1): 155-159

Čuk I, Fink H, Leskošek B. Modeling the final score in artistic gymnastics by different weights of difficulty and execution. Sci Gym J, 2012; 4(1): 73-82

Čuk I, Forbes W. How apparatus difficulty scores affect all around results in men's artistic gymnastics. Sci Gym J, 2010; 2(3): 57-63

Dallas G, Kirialanis P. Judges' evaluation of routines in men's artistic gymnastics. Sci Gym J, 2010; 2(2): 49-58

Fédération Internationale de Gymnastique. 2017 Code of Points. Men's Artistic Gymnastics. Lausanne: FIG; 2017

Fédération Internationale de Gymnastique. 2013 Code of Points. Men's Artistic Gymnastics. Lausanne: FIG; 2013

Heinen T, Vinken PM, Velentzas K. Judging performance in gymnastics: A matter of motor or visual experience?. Sci Gym J, 2012; 4(1): 63-72 
Leskošek B, Čuk I, Bučar Pajek M. Trends in E and D scores and their influence on final results of male gymnasts at European Championships 2005-2011. Sci Gym J, 2013; 5(1): 29-38

Leskošek B, Čuk I, Karácsony I, Pajek J, Bučar M. Reliability and validity of judging in men's artistic gymnastics at the 2009 University Games. Sci Gym J, 2010; 2(1): 25-34

Leskošek B, Čuk I, Pajek J, Forbes W, Bučar-Pajek M. Bias of judging in men's artistic gymnastics at the European Championships 2011. Biol Sport, 2012; 29(2): 107-113

Marinšek M, Čuk I. Landing errors in the men's floor exercise are caused by flight characteristics. Biol Sport, 2010; 27(2): 123-128

Pizzera A. Gymnastic judges benefit from their own motor experience as gymnasts. Res $Q$ Exercise Sport, 2012; 83(4): 603-607

Plessner H, Schallies E. Judging the cross on rings: A matter of achieving shape consistency. Appl Cognitive Psych, 2005; 19: 1145-1156

Plessner H. Expectation biases in gymnastics judging. J Sport Exercise Psy, 1999; 21(2): 131-144.

Ste-Marie DM. Expertise in women's gymnastic judging: An observational approach. Percept Motor Skill, 2000; 90: 543-546

\section{Corresponding author:}

\section{Jonas Rohleder}

Institute of Professional Sport Education and Sport Qualifications, German Sport University Cologne, Am Sportpark Müngersdorf 6, 50933 Cologne, Germany.

Telephone number: +4922149824160

E-mail: j.rohleder@dshs-koeln.de 